\title{
Asymptotic dynamics of the Leslie-Gower competition system with Allee effects and stocking
}

Yunshyong Chow ${ }^{1}$ and Sophia R-J Jang ${ }^{2 *}$

"Correspondence: srjjang@gmail.com

2Department of Mathematics and Statistics, Texas Tech University, Lubbock, TX 79409-1042, USA Full list of author information is available at the end of the article

\begin{abstract}
We investigate the classical Leslie-Gower competition system where one of the two competing populations is subject to Allee effects and is also under constant stocking. The model can have either no interior steady state, a unique interior steady state, two interior steady states or three interior steady states depending on parameter values. Using the tools of monotone planar systems, we provide basins of attraction for the local attractors and for the non-hyperbolic steady states. It is concluded that stocking of the weaker competitor can promote the coexistence of both competing populations.
\end{abstract}

Keywords: competition system; stable manifold; Allee effects; stocking

\section{Introduction}

The Allee effect, referring to the reduced fitness or the decline in population growth at low population densities or sizes, was first observed by Allee [1]. It has significant impact on population survival when the population is at low level. There has been a rebound of interest in Allee effects recently due to fragmentation of habitats, invasions of exotic species, biological control of pest, etc. We refer the reader to [2] and references cited therein for biological motivation and mathematical models of Allee effects.

When a population is subject to Allee effects, it is well known that there exists a critical population level below which the population will go extinct [2]. Therefore, such a population is in danger of extinction if its population size or density is small. Frequently, constant levels of the external populations are added to the resident populations per generation in order to conserve the endangered populations [3]. Motivated by this, we consider the classical two-dimensional Leslie-Gower competition system where one of the populations is subject to Allee effects and is also under constant stocking. Several discrete-time mathematical models have been proposed to study the effects of external stocking. See AlSharawi and Rhouma [4], Elaydi and Yakubu [5], Kulenović and Nurkanović [6] and references cited therein.

The authors in [6] investigate the Leslie-Gower competition system when one of the two populations is under constant stocking. The resulting model has at most two interior steady states and the basins of attraction of the steady states are completely determined [6]. Unlike the model studied in [6], we assume in this study that one of the two competing populations is subject to Allee effects and is also under constant stocking. Using the 
tools of two-dimensional monotone maps $[7,8]$, we provide the asymptotic dynamics of the resulting system. The model with no stocking has been studied in [9]; it can have at most two interior steady states. It is proved in [9] that one of the interior steady states is a saddle point and its global stable manifold is the boundary for population coexistence and competitive exclusion. With stocking, it is shown in the present study that the model can have up to three interior steady states, one of which is a saddle point and the other two are local attractors. The global stable manifold of the saddle point separates the positive cone into two positively invariant regions such that the solutions converge to either one of the interior steady states for all positive initial populations. As a consequence, stocking of one of the two competing populations with Allee effects can promote population coexistence.

In the following section, we present the model and its preliminary analysis. The global behavior of the solutions is studied in Section 3. The final section provides discussion and conclusions.

\section{The model and preliminary results}

Let $x(t)$ and $y(t)$ denote two competing populations at time $t=0,1, \ldots$. The interaction between populations $x$ and $y$ proposed by Leslie and Gower $[10,11]$ is described by the following system:

$$
\left\{\begin{array}{l}
x(t+1)=\frac{\lambda_{1} x(t)}{1+x(t)+c_{1} y(t)}, \\
y(t+1)=\frac{\lambda_{2} y(t)}{1+c_{2} x(t)+y(t)},
\end{array}\right.
$$

where the model has been rescaled to reduce the number of parameters. The parameters $c_{1}$ and $c_{2}$ are the competition coefficients and $\lambda_{1}$ and $\lambda_{2}$ are the intrinsic growth rates. These parameters are positive constants. The asymptotic dynamics of (2.1) is well studied by Cushing et al. [10]. Under some constraints on the parameters, the model has at most one interior steady state and the dynamics is similar to the classical Lotka-Volterra competition system of ordinary differential equations [10].

Kulenović and Nurkanović [6] recently studied the following Leslie-Gower competition model with stocking:

$$
\left\{\begin{array}{l}
x(t+1)=\frac{\lambda_{1} x(t)}{1+x(t)+c_{1} y(t)}+h, \\
y(t+1)=\frac{\lambda_{2} y(t)}{1+c_{2} x(t)+y(t)}
\end{array}\right.
$$

where $h>0$ is the constant stocking. It is proved in [6] that (2.2) has at most two interior steady states and the basins of attractions for the steady states are explicitly provided.

In [9], Jang proposes the following competition system with Allee effects occurring in the $x$ population:

$$
\left\{\begin{array}{l}
x(t+1)=\left(\frac{\lambda_{1} x(t)}{1+x(t)+c_{1} y(t)}\right)\left(\frac{x(t)}{m+x(t)}\right), \\
y(t+1)=\frac{\lambda_{2} y(t)}{1+c_{2} x(t)+y(t)} .
\end{array}\right.
$$

The expression $\frac{x}{m+x}$ models the mechanism of Allee effects for population $x$. Individuals within the population have to find others to reproduce or to exploit resources. The fraction 
$\frac{x}{m+x}$ is the probability of an individual successfully finding a mate or a cooperative individual when population size is $x$. The larger $m$ the smaller this probability is, and therefore $1 / m$ may be interpreted as an individual?s searching efficiency 12]. The resulting system can have two interior steady states in which one of them is a saddle point. The global stable manifold of the saddle point is the boundary for population coexistence and competitive exclusion [9].

Motivated by the work in [6] and for the conservation of endangered populations, we propose the following competition system with Allee effects and stocking occurring in the $x$ population:

$$
\left\{\begin{array}{l}
x(t+1)=\left(\frac{\lambda_{1} x(t)}{1+x(t)+c_{1} y(t)}\right)\left(\frac{x(t)}{m+x(t)}\right)+u, \\
y(t+1)=\frac{\lambda_{2} y(t)}{1+c_{2} x(t)+y(t)}
\end{array}\right.
$$

with nonnegative initial conditions, where $u>0$ denotes the constant stocking and the other parameters have the same biological meanings as those parameters in (2.3).

If $y(0)=0$, then $y(t)=0$ for $t>0$ and (2.4) reduces to the following scalar equation:

$$
x(t+1)=\left(\frac{\lambda_{1} x(t)}{1+x(t)}\right)\left(\frac{x(t)}{m+x(t)}\right)+u .
$$

Let

$$
f(x)=\left(\frac{\lambda_{1} x}{1+x}\right)\left(\frac{x}{m+x}\right)+u .
$$

Then $f(0)=u, f^{\prime}(x)>0$ for $x>0$ and $\lim _{x \rightarrow \infty} f(x)=\lambda_{1}+u$. An interior steady state $x$ of (2.5) satisfies $x^{3}+a_{1} x^{2}+a_{2} x+a_{3}=0$, where $a_{1}=m+1-u-\lambda_{1}, a_{2}=m-u(m+1)$, and $a_{3}=-u m$. By the Descartes rule of signs [13], the interior steady state is unique if either (a): $a_{i}>0$, $i=1,2$, (b): $a_{1}>0$ and $a_{2}<0$, or (c): $a_{i}<0, i=1,2$ holds. On the other hand if (d): $a_{1}<0$ and $a_{2}>0$ holds, then (2.5) may have three interior steady states. In the following analysis we assume that (2.5) has a unique steady state $\bar{x}$, where $u<\bar{x}<u+\lambda_{1}$. Notice $f^{\prime}(\bar{x})<1$ and since $f$ is monotone increasing, we have the following asymptotic dynamics for (2.5).

Proposition 2.1 Equation (2.5) has a unique steady state $\bar{x}$, where $u<\bar{x}<u+\lambda_{1}$ and $\bar{x}$ is globally asymptotically stable in $[0, \infty)$ for $(2.5)$.

When (2.5) has three interior steady states, then the middle steady state is a repeller while the other two interior steady states are asymptotically stable (cf. [14, Theorem 2.1]). Similar to the proof in [14], the asymptotic dynamics of (2.5) can be completely determined when there are three interior steady states. However, we assume (2.5) has a unique interior steady state for simplicity. The analysis carried out in this work can be applied to the case where (2.5) has three interior steady states as well.

To study system (2.4), we first notice that (2.4) always has the steady state $E_{0}=(\bar{x}, 0)$, where the $y$ population is extinct. Moreover, $E_{0}$ is the only steady state on the boundary. If $\lambda_{2} \leq 1$, then $\lim _{t \rightarrow \infty} y(t)=0$ and all solutions of (2.4) converge to the steady state $E_{0}$. Therefore, we assume 
for the remaining discussion. Let $F(x, y)=\left(f_{1}(x, y), f_{2}(x, y)\right)$ be the map induced by $(2.4)$, where

$$
f_{1}(x, y)=\left(\frac{\lambda_{1} x}{1+x+c_{1} y}\right)\left(\frac{x}{m+x}\right)+u, \quad f_{2}(x, y)=\frac{\lambda_{2} y}{1+c_{2} x+y} .
$$

The Jacobian matrix $J$ of $(2.4)$ evaluated at $(x, y)$ is given by

$$
J(x, y)=\left(\begin{array}{ll}
\frac{\partial f_{1}}{\partial x} & \frac{\partial f_{1}}{\partial y} \\
\frac{\partial f_{2}}{\partial x} & \frac{\partial f_{2}}{\partial y}
\end{array}\right)
$$

with

$$
\begin{aligned}
\frac{\partial f_{1}}{\partial x} & =\frac{\lambda_{1}\left(1+c_{1} y\right) x}{\left(1+x+c_{1} y\right)^{2}(m+x)}+\frac{\lambda_{1} m x}{\left(1+x+c_{1} y\right)(m+x)^{2}}, \\
\frac{\partial f_{1}}{\partial y} & =\left(\frac{-\lambda_{1} c_{1} x}{\left(1+x+c_{1} y\right)^{2}}\right)\left(\frac{x}{m+x}\right), \\
\frac{\partial f_{2}}{\partial x} & =\frac{-\lambda_{2} c_{2} y}{\left(1+c_{2} x+y\right)^{2}} \quad \text { and } \quad \frac{\partial f_{2}}{\partial y}=\frac{\lambda_{2}\left(1+c_{2} x\right)}{\left(1+c_{2} x+y\right)^{2}} .
\end{aligned}
$$

After some calculations, the determinant of the Jacobian matrix $J(x, y)$, det $J(x, y)$, can be written as

$$
\operatorname{det} J(x, y)=\frac{\lambda_{1} \lambda_{2} x\left(1+c_{2} x+c_{1} y\right)}{\left(1+x+c_{1} y\right)^{2}\left(1+c_{2} x+y\right)^{2}(m+x)}+\frac{\lambda_{1} \lambda_{2} m x\left(1+c_{2} x\right)}{\left(1+x+c_{1} y\right)\left(1+c_{2} x+y\right)^{2}(m+x)^{2}} .
$$

It follows that

$$
\operatorname{det} J(x, y)>0 \text { for } x>0 \text { and } y \geq 0 \text {. }
$$

Let $\mathbb{R}_{+}^{2}=\left\{(x, y) \in \mathbb{R}^{2}: x \geq 0, y \geq 0\right\}$ and let int $\left(\mathbb{R}_{+}^{2}\right)$ denote the interior of $\mathbb{R}_{+}^{2}$. It is straightforward to verify that solutions of (2.4) remain nonnegative and are bounded for $t>0$, and the map $F$ is one-to-one on $\mathbb{R}_{+}^{2}$.

Proposition 2.2 Solutions of (2.4) remain nonnegative and are bounded for $t>0$. Moreover, the map $F$ is one-to-one on $\mathbb{R}_{+}^{2}$.

Proof Let $\left(x_{0}, y_{0}\right)$ and $\left(x_{1}, y_{1}\right)$ in $\mathbb{R}_{+}^{2}$ be such that $F\left(x_{0}, y_{0}\right)=F\left(x_{1}, y_{1}\right)$, i.e., $f_{i}\left(x_{0}, y_{0}\right)=f_{i}\left(x_{1}, y_{1}\right)$, $i=1$, 2. If $x_{0}=0$ or $y_{0}=0$, then $\left(x_{0}, y_{0}\right)=\left(x_{1}, y_{1}\right)$ holds trivially. We now assume $x_{i}, y_{i}>0$ for $i=0,1$ and $x_{0}<x_{1}$. Then $y_{0}<y_{1}$ since $f_{2}\left(x_{0}, y_{0}\right)=f_{2}\left(x_{1}, y_{1}\right)$. Let $Y_{i}=Y_{i}(x)$ be defined by $f_{i}\left(x, Y_{i}(x)\right)=f_{i}\left(x_{0}, y_{0}\right)$ for $i=1,2$. Then $y=Y_{1}(x)$ and $y=Y_{2}(x)$ have two positive intersections $\left(x_{0}, y_{0}\right)$ and $\left(x_{1}, y_{1}\right)$. It is easy to see that $y=Y_{2}(x)$ is a line with $Y_{2}^{\prime}(x)=\frac{c_{2} y_{0}}{1+c_{2} x_{0}}>0$, and $Y_{1}^{\prime}(x)>0$ and $Y_{1}^{\prime \prime}(x)=\frac{2 \alpha m^{2}}{(m+x)^{3}}>0$ for $x>0$, where $\alpha=\frac{\left(m+x_{0}\right)\left(1+c_{0}+c_{1} y_{0}\right)}{x_{0}^{2}}$. Moreover, a simple calculation yields $Y_{1}^{\prime}(x)-Y_{2}^{\prime}(x)=\frac{\operatorname{det} J(x, y)}{-\frac{\partial f_{1}, \frac{\partial}{\partial}}{\partial y}}>0$ by $(2.9)$. Hence $Y_{1}(x)>Y_{2}(x)$ on $\left(x_{0}, \infty\right)$ and we obtain a contradiction. It is possible that $y=Y_{1}(x)$ and $y=Y_{2}(x)$ have two solutions, but at most one positive solution. Hence $F$ is one-to-one on $\mathbb{R}_{+}^{2}$.

Define the partial ordering $? \leq_{K}$ ? on $\mathbb{R}_{+}^{2}$ by $X_{1}=\left(x_{1}, y_{1}\right) \leq_{K} X_{2}=\left(x_{2}, y_{2}\right)$ if $x_{1} \leq x_{2}$ and $y_{1} \geq y_{2}, X_{1}<_{K} X_{2}$ if $X_{1} \leq_{K} X_{2}$ and $X_{1} \neq X_{2}$, and $X_{1} \ll_{K} X_{2}$ if $x_{1}<x_{2}$ and $y_{1}>y_{2}$. A map 
$\mathbf{T}: \mathbb{R}_{+}^{2} \rightarrow \mathbb{R}_{+}^{2}$ is said to be monotone with respect to $\leq_{K}$ if $\mathbf{T}\left(x_{1}, y_{1}\right) \leq_{K} \mathbf{T}\left(x_{2}, y_{2}\right)$ whenever $\left(x_{1}, y_{1}\right) \leq_{K}\left(x_{2}, y_{2}\right)$ and $\mathbf{T}$ is strongly monotone if $\mathbf{T}\left(x_{1}, y_{1}\right) \ll_{K} \mathbf{T}\left(x_{2}, y_{2}\right)$ whenever $\left(x_{1}, y_{1}\right)<_{K}$ $\left(x_{2}, y_{2}\right)$.

A subset $B$ of $\mathbb{R}^{2}$ is $K$-convex with respect to $\leq_{K}$ if $B$ contains the line segment joining any of its two points that are ordered by $\leq_{K}$ [8]. An $n \times n$ matrix $P=\left(p_{i j}\right)$ is $K$-positive on a subset $C$ of $\mathbb{R}_{+}^{2}$ if its diagonal entries are nonnegative on $C$ and its off diagonal entries are nonpositive on $C$ [8]. We refer the reader to Smith [8] for the general theory of twodimensional monotone maps.

Since populations $x$ and $y$ engage in interspecific competition, using the tool of monotone planar maps, the asymptotic dynamics of (2.4) can easily be understood as shown below.

Theorem 2.3 The map F is monotone on $\mathbb{R}_{+}^{2}$ and strongly monotone on $\operatorname{int}\left(\mathbb{R}_{+}^{2}\right)$. Moreover, every solution of (2.4) converges to a steady state.

Proof Let $X_{i}(t)=\left(x_{i}(t), y_{i}(t)\right), i=1,2$, be two solutions of (2.4) with $X_{1}(0) \leq_{K} X_{2}(0)$. Then $x_{1}(1) \leq f_{1}\left(x_{2}(0), y_{1}(0)\right) \leq f_{1}\left(x_{2}(0), y_{2}(0)\right)=x_{2}(1)$ and $y_{1}(1) \geq f_{2}\left(x_{2}(0), y_{1}(0)\right) \geq f_{2}\left(x_{2}(0)\right.$, $\left.y_{2}(0)\right)=y_{2}(1)$, i.e., $X_{1}(1) \leq_{K} X_{2}(1)$. Inductively, $X_{1}(t) \leq_{K} X_{2}(t)$ for $t \geq 1$ and $F$ is monotone on $\mathbb{R}_{+}^{2}$ with respect to $\leq_{K}$. Similarly, one can prove that $F$ is strongly monotone on $\operatorname{int}\left(\mathbb{R}_{+}^{2}\right)$. Notice $\operatorname{int}\left(\mathbb{R}_{+}^{2}\right)$ is forwardly invariant under $F$, contains order intervals and is $\leq_{K^{-}}$ convex. The determinant of the Jacobian matrix of (2.4) is positive on $\operatorname{int}\left(\mathbb{R}_{+}^{2}\right)$ by (2.9). Moreover, the Jacobian matrix is $K$-positive in $\operatorname{int}\left(\mathbb{R}_{+}^{2}\right)$ by $(2.8)$ and the map $F$ is one-to-one by Proposition 2.2. Therefore, conditions $(\mathrm{H}+)$ given in [8] are satisfied for $F$ on $\operatorname{int}\left(\mathbb{R}_{+}^{2}\right)$. It follows from [8, Theorem 4.2, Lemma 4.3] that solutions of (2.4) with initial conditions in $\operatorname{int}\left(\mathbb{R}_{+}^{2}\right)$ are eventually componentwise monotone and hence are convergent. If $y(0)=0$ and $x(0) \geq 0$, then the solution converges to $(\bar{x}, 0)$. If $x(0)=0$ and $y(0)>0$, then $x(1)=u$ and $y(1)>0$, i.e., solutions start on the positive $y$-axis will be mapped into $\operatorname{int}\left(\mathbb{R}_{+}^{2}\right)$ in one iteration. We conclude that every solution of (2.4) converges to a steady state.

Let $\left(x_{0}, y_{0}\right) \in \mathbb{R}_{+}^{2}$ be given. Relative to $\left(x_{0}, y_{0}\right)$, we divide $\mathbb{R}_{+}^{2}$ into four quadrants in a counter-clockwise manner: $Q_{1}\left(x_{0}, y_{0}\right)=\left\{(x, y) \in \mathbb{R}_{+}^{2}: x \geq x_{0}, y \geq y_{0}\right\}, Q_{2}\left(x_{0}, y_{0}\right)=\{(x, y) \in$ $\left.\mathbb{R}_{+}^{2}: x \leq x_{0}, y \geq y_{0}\right\}$, and $Q_{3}\left(x_{0}, y_{0}\right)$ and $Q_{4}\left(x_{0}, y_{0}\right)$ are defined similarly. If $\left(x_{0}, y_{0}\right)$ is a steady state of (2.4), then it is easy to see that $Q_{i}\left(x_{0}, y_{0}\right), i=2,4$, are positively invariant since $F$ is monotone with respect to $\leq_{K}$. Indeed, let $\left(x_{0}, y_{0}\right)$ be a steady state of system (2.4) and let $(x, y) \in Q_{2}\left(x_{0}, y_{0}\right)$, i.e., $(x, y) \leq_{K}\left(x_{0}, y_{0}\right)$. Then since $F$ is monotone with respect to $\leq_{K}$, we obtain $F^{t}(x, y) \leq_{K} F^{t}\left(x_{0}, y_{0}\right)=\left(x_{0}, y_{0}\right)$ for $t=1,2, \ldots$. Hence $F^{t}(x, y) \in Q_{2}\left(x_{0}, y_{0}\right)$ for $t=1,2, \ldots$ and $Q_{2}\left(x_{0}, y_{0}\right)$ is positively invariant. The proof of positive invariance of $Q_{4}\left(x_{0}, y_{0}\right)$ is similar.

The Jacobian matrix of $(2.4)$ evaluated at $E_{0}=(\bar{x}, 0)$ is given by

$$
J(\bar{x}, 0)=\left(\begin{array}{cc}
f^{\prime}(\bar{x}) & * \\
0 & \frac{\lambda_{2}}{1+c_{2} \bar{x}}
\end{array}\right),
$$

where $*$ is an unimportant term. It follows from (2.10) that $E_{0}$ is locally asymptotically stable if $\frac{\lambda_{2}}{1+c_{2} \bar{x}}<1$ and unstable if $\frac{\lambda_{2}}{1+c_{2} \bar{x}}>1$. 
Remark 1 When $\frac{\lambda_{2}-1}{c_{2}}=\bar{x}$, then $E_{0}$ is non-hyperbolic with a stable manifold lying on the nonnegative $x$-axis by (2.10). One may use the technique of center manifold [15] to determine the stability of $E_{0}$. However, we do not consider this critical case in the present investigation.

To discuss the existence of an interior steady state, we set $x=f_{1}(x, y)$ and obtain the following $x$-nullcline:

$$
y=q_{1}(x)=\frac{1}{c_{1}}\left(\frac{\lambda_{1} x^{2}}{(m+x)(x-u)}-x-1\right), \quad x>u .
$$

Notice $\lim _{x \rightarrow u^{+}} q_{1}(x)=\infty, q_{1}(\bar{x})=0$, where $\bar{x}$ is the unique steady state of $(2.5)$ and

$$
\begin{aligned}
& q_{1}^{\prime}(x)=\frac{1}{c_{1}}\left(\frac{\lambda_{1} x((m-u) x-2 u m)}{(x-u)^{2}(m+x)^{2}}-1\right), \quad x>u, \\
& q_{1}^{\prime \prime}(x)=\frac{2 \lambda_{1}}{c_{1}}\left(\frac{(u-m) x^{3}+3 m u x^{2}+u^{2} m^{2}}{(x-u)^{3}(m+x)^{3}}\right), \quad x>u .
\end{aligned}
$$

Moreover,

$$
q_{1}(x)>0 \quad \text { if and only if } \quad x \in(u, \bar{x}) .
$$

Similarly, letting $y=f_{2}(x, y)$ and assuming $y \neq 0$, we have the following non-trivial $y$ nullcline:

$$
y=\hat{q}_{2}(x)=\lambda_{2}-1-c_{2} x
$$

Notice that $y=\hat{q}_{2}(x)$ is a straight line with $y$-intercept $\lambda_{2}-1$ and $x$-intercept $\frac{\lambda_{2}-1}{c_{2}}$. Let $q_{2}(y)$ denote the inverse function of $\hat{q}_{2}(x)$. A positive intersection $x^{*}$ of the two nullclines will result in an interior steady state $\left(x^{*}, y^{*}\right)$ only when $x^{*}<\min \left\{\frac{\lambda_{2}-1}{c_{2}}, \bar{x}\right\}$, where $y^{*}=\lambda_{2}-1-c_{2} x^{*}$. If $\left(x_{0}, y_{0}\right)$ is an interior steady state, then the Jacobian matrix of (2.4) evaluated at $\left(x_{0}, y_{0}\right)$ can be rewritten as

$$
\left(\begin{array}{ll}
a & b \\
c & d
\end{array}\right),
$$

where $a, d>0, b, c<0$ and their values depend on $\left(x_{0}, y_{0}\right)$. The characteristic polynomial $p(\lambda)$ of $J\left(x_{0}, y_{0}\right)$ is

$$
p(\lambda)=\lambda^{2}-(a+d) \lambda+(a d-b c)
$$

with discriminant

$$
(a+d)^{2}-4(a d-b c)=(a-d)^{2}+4 b c>0
$$

Therefore, eigenvalues of $J\left(x_{0}, y_{0}\right)$ are positive real numbers by (2.9). We conclude that $\left(x_{0}, y_{0}\right)$ is a saddle point if $p(1)<0$, and $\left(x_{0}, y_{0}\right)$ is locally asymptotically stable if $p(1)>0$ 
and $\operatorname{det} J\left(x_{0}, y_{0}\right)=a d-b c<1$. This observation will be useful to the analysis presented in Section 3.

Notice that at any interior steady state $\left(x_{0}, y_{0}\right)$, we always have

$$
d=\frac{\lambda_{2}\left(1+c_{2} x_{0}\right)}{\left(1+c_{2} x_{0}+y_{0}\right)^{2}}<\frac{\lambda_{2}}{1+c_{2} x_{0}+y_{0}}=1 \text {. }
$$

Moreover, differentiating $x=f_{1}\left(x, q_{1}(x)\right)$ with respect to $x$ yields

$$
q_{1}^{\prime}(x)=\frac{1-a}{b},
$$

and differentiating $y=f_{2}\left(q_{2}(y), y\right)$ with respect to $y$, one obtains

$$
q_{2}^{\prime}(y)=\frac{1-d}{c} .
$$

Using equalities (2.17)-(2.18), we have

$$
p(1)=(1-a)(1-d)-b c=\left(q_{1}^{\prime}(x) q_{2}^{\prime}(y)-1\right) b c .
$$

As $b c>0$, the sign of $p(1)$ then depends on $q_{1}^{\prime}(x) q_{2}^{\prime}(y)-1$.

\section{Asymptotic dynamics}

In this section, we study the existence of interior steady states and investigate the asymptotic dynamics of system (2.4). Observe that if $\frac{\lambda_{2}-1}{c_{2}} \leq u$, then the two non-trivial nullclines cannot have any positive intersections and (2.4) has no interior steady state. Moreover, $E_{0}=(\bar{x}, 0)$ is locally asymptotically stable by $(2.10)$ since $u<\bar{x}$ and therefore $E_{0}$ is globally asymptotically stable by Theorem 2.3 . The proof of the following theorem is omitted.

Theorem 3.1 If $\frac{\lambda_{2}-1}{c_{2}} \leq u$, then $E_{0}=(\bar{x}, 0)$ is globally asymptotically stable in $\mathbb{R}_{+}^{2}$ for (2.4).

It follows from Theorem 3.1 that if the stocking $u$ of the $x$ population is large or if the $x$ population is more competitive with $c_{2} \geq \frac{\lambda_{2}-1}{u}$, then the $x$ population will drive the $y$ population to extinction even when the $x$ population is subject to Allee effects. In the remaining discussion, we assume that the following inequality holds:

$$
\frac{\lambda_{2}-1}{c_{2}}>u
$$

Let

$$
\Gamma=\left\{(x, y) \in \mathbb{R}_{+}^{2}: y>0\right\}
$$

and let $W_{i}^{s}$ be the stable manifold of the steady state termed $E_{i}^{*}$. The global stable manifold of a steady state $E^{*}$ is denoted by $W^{s}$. If $R$ is a subset of $\mathbb{R}_{+}^{2}$ and $E$ is a steady state in $R$, we say that $E$ is globally asymptotically stable (GAS in abbreviation) in $R$ if $E$ is locally asymptotically stable and globally attracting in $R$.

To simplify the statements of our analysis, we let cases 1-6 summarized in Table 1 to denote the different global asymptotic behavior of system (2.4), which will be used in the 
Table 1 Dynamics of system (2.4)

\begin{tabular}{|c|c|}
\hline Cases & Global dynamical behavior of (2.4) \\
\hline 1 & (2.4) has no interior steady state and $E_{0}$ is GAS in $\mathbb{R}_{+}^{2}$. \\
\hline 2 & (2.4) has a unique interior steady state $E^{*}$ which is GAS in $\Gamma$. \\
\hline 3 & $\begin{array}{l}\text { (2.4) has a unique interior steady state } E^{*} \text { which is non-hyperbolic. Ws } \\
\text { separates } \mathbb{R}_{+}^{2} \text { into two positively invariant regions } R_{1} \text { and } R_{2} \text { such that } E_{0} \text { is GAS } \\
\text { in } R_{2} \text { and solutions with initial conditions in } R_{1} \text { converge to } E^{*} \text {. }\end{array}$ \\
\hline 4 & $\begin{array}{l}\text { (2.4) has two hyperbolic interior steady states } E_{1}^{*} \text { and } E_{2}^{*} \cdot W_{2}^{s} \text { separates } \mathbb{R}_{+}^{2} \text { into } \\
\text { two positively invariant regions } R_{1} \text { and } R_{2} \text { such that } E_{1}^{*} \text { and } E_{0} \text { are GAS in } R_{1} \\
\text { and } R_{2} \text {, respectively. }\end{array}$ \\
\hline 5 & $\begin{array}{l}\text { (2.4) has two interior steady states, one is hyperbolic and the other is } \\
\text { non-hyperbolic. The global stable manifold of the non-hyperbolic steady state } \\
\text { separates } \mathbb{R}_{+}^{2} \text { into two positively invariant regions } R_{1} \text { and } R_{2} \text { such that the } \\
\text { other interior steady state is GAS in } R_{1} \text { and } E_{0} \text { is GAS in } R_{2} \text {. }\end{array}$ \\
\hline 6 & $\begin{array}{l}\text { (2.4) has three interior steady states } E_{i}^{*}, i=1,2,3 . W_{2}^{s} \text { separates } \mathbb{R}_{+}^{2} \text { into two } \\
\text { positively invariant regions } R_{1} \text { and } R_{2} \text { such that } E_{1}^{*} \text { and } E_{3}^{*} \text { are GAS in } R_{1} \text { and } R_{2} \text {, } \\
\text { respectively. }\end{array}$ \\
\hline
\end{tabular}

statements of the remaining theorems. We remark that the positively invariant regions $R_{1}$ and $R_{2}$ given in cases 3-6 are in general not the same. Moreover, coexistence of both competing populations can occur in cases 2 and 6 . There exists a unique globally attracting coexisting steady state for case 2 . There are three interior steady states in case 6 and the magnitudes of the steady state where the populations converge will then depend on initial populations.

If $m \leq u$, then the $x$-nullcline $y=q_{1}(x)$ is strictly decreasing on $(u, \infty)$ by $(2.12)$. In this case, the two nullclines can have either no positive intersections, two positive intersections or one positive intersection depending on the parameter values. Indeed, the $x$-nullcline $y=q_{1}(x)$ separates $\mathbb{R}_{+}^{2}$ into two regions:

$$
A_{1}=\left\{(x, y) \in \mathbb{R}_{+}^{2}: y<q_{1}(x)\right\}, \quad A_{2}=\left\{(x, y) \in \mathbb{R}_{+}^{2}: y>q_{1}(x)\right\} .
$$

Similarly, the curve $x=q_{2}(y)$ separates $\mathbb{R}_{+}^{2}$ into two subsets:

$$
B_{1}=\left\{(x, y) \in \mathbb{R}_{+}^{2}: x<q_{2}(y)\right\}, \quad B_{2}=\left\{(x, y) \in \mathbb{R}_{+}^{2}: x>q_{2}(y)\right\} .
$$

If $B_{1} \cap A_{2} \neq \emptyset$, then the two nullclines have two positive intersections and (2.4) has two interior steady states. If $B_{1} \cap A_{2}=\emptyset$ and $y=\hat{q}_{2}(x)$ is not tangent to $y=q_{1}(x)$, then the two nullclines have no positive intersections and thus (2.4) has no interior steady state. If $B_{1} \cap A_{2}=\emptyset$ and $y=\hat{q}_{2}(x)$ is tangent to $y=q_{1}(x)$ at $x^{*}$, where $u<x^{*}<\frac{\lambda_{2}-1}{c_{2}}$, then (2.4) has a unique interior steady state. The asymptotic dynamics of (2.4) is summarized below for $m \leq u$.

Theorem 3.2 Let $\frac{\lambda_{2}-1}{c_{2}}>u$ and $m \leq u$. The following statements hold for (2.4).

(a) If $\frac{\lambda_{2}-1}{c_{2}}>\bar{x}$, then case 2 holds.

(b) Let $\frac{\lambda_{2}-1}{c_{2}}<\bar{x}$. Then $E_{0}$ is locally asymptotically stable.

(i) If $B_{1} \cap A_{2}=\emptyset$ and $y=\hat{q}_{2}(x)$ is not tangent to $y=q_{1}(x)$, then case 1 holds.

(ii) If $B_{1} \cap A_{2}=\emptyset$ and $y=\hat{q}_{2}(x)$ is tangent to $y=q_{1}(x)$ at $x^{*}$, where $u<x^{*}<\frac{\lambda_{2}-1}{c_{2}}$, then case 3 holds.

(iii) If $B_{1} \cap A_{2} \neq \emptyset$, then case 4 holds. 
Proof (a) Since $\frac{\lambda_{2}-1}{c_{2}}>\bar{x}$ and both nullclines are strictly decreasing, it can easily be shown that the two nullclines have a unique positive intersection $x^{*}$, where $u<x^{*}<\bar{x}$. Indeed, let $q(x)=q_{1}(x)-\hat{q}_{2}(x)$, the difference of the two isoclines. Then $q^{\prime \prime}(x)=q_{1}^{\prime \prime}(x)>0$ for $x>u$ by (2.12) and the assumption $u \geq m$. It is clear that $q(x)=0$ has at least one positive solution in $(u, \bar{x})$ since $\lim _{x \rightarrow u^{+}} q(x)=\infty$ and $q(\bar{x})<0$. If $q(x)=0$ has two solutions $x_{i}, i=1,2$, where $u<$ $x_{1}<x_{2}<\bar{x}$, then $q(\bar{x})<0$ implies that either $q(x)=0$ has at least one more solution in $\left(x_{2}, \bar{x}\right)$ or $q^{\prime}\left(x_{i}\right)=0$ for some $i=1,2$. In either of these cases, we obtain a contradiction to $q^{\prime \prime}(x)>0$ on $(u, \infty)$. Therefore, $q(x)=0$ cannot have more than one positive solution in $(u, \bar{x})$ and (2.4) has a unique interior steady state $E^{*}=\left(x^{*}, y^{*}\right)$. We can easily verify that $E^{*}$ is locally asymptotically stable. Indeed, since $q_{1}^{\prime}\left(x^{*}\right)<0, q_{2}^{\prime}\left(y^{*}\right)<0$, and $b, c<0$, we have from (2.16)(2.17) that $a<1$ and $d<1$. Hence $\operatorname{det} J\left(E^{*}\right)=a d-b c<a d<1$. Observe that $q_{1}^{\prime}\left(x^{*}\right)<-c_{2}$, i.e., $\frac{q_{1}^{\prime}\left(x^{*}\right)}{-c_{2}}>1$ and hence $q_{1}^{\prime}\left(x^{*}\right) q_{2}^{\prime}\left(y^{*}\right)-1>0$, i.e., $p(1)>0$ by (2.19). Therefore, $E^{*}$ is locally asymptotically stable by the Jury conditions. On the other hand, $E_{0}$ is a saddle point with stable manifold being the nonnegative $x$-axis by (2.10). It follows from Theorem 2.3 that solutions with initial conditions in $\Gamma$ converge to $E^{*}$ and hence $E^{*}$ is globally asymptotically stable in $\Gamma$, i.e., case 2 holds.

(b) By the assumption and (2.10), $E_{0}$ is locally asymptotically stable. The proof of (i) is trivial by Theorem 2.3 since there is no interior steady state. To prove (ii), observe that the straight line $y=\hat{q}_{2}(x)$ is tangent to the curve $y=q_{1}(x)$ at $x^{*}$ in which $E^{*}=\left(x^{*}, y^{*}\right)$ is the unique interior steady state. Thus, $q_{1}^{\prime}\left(x^{*}\right)=-c_{2}$ and $q_{1}^{\prime}\left(x^{*}\right) q_{2}^{\prime}\left(y^{*}\right)=1$. Therefore, $p(1)=0$ by (2.19) and $E^{*}$ is non-hyperbolic. The other eigenvalue of $J\left(E^{*}\right)$ is $\operatorname{det} J\left(E^{*}\right)=a d-b c<a d<$ 1 since $q_{1}$ is decreasing implying $a<1$. Moreover, eigenvectors of $J\left(E^{*}\right)$ with respect to $\operatorname{det} J\left(E^{*}\right)$ are not parallel to the coordinate axes. Theorems 1-4 of [7] imply that the stable manifold $W^{s}$ of $E^{*}$ is $C^{1}$ and strictly increasing. Since (2.4) has no 2-cycle solutions and no other interior steady state, $W^{s}$ intersects the nonnegative $y$-axis and is unbounded in $Q_{1}\left(E^{*}\right)$ by Corollary 2 of [7]. Therefore, Theorem 6 of [7] implies that $W^{s}$ separates $\mathbb{R}_{+}^{2}$ into two positively invariant regions $R_{1}$ and $R_{2}$ with $E_{0} \in R_{2}$. Since every solution converges to a steady state by Theorem 2.3 and $R_{i}$ is forwardly invariant, solutions with initial conditions in $R_{1}$ converge to $E^{*}$. Observe that $\hat{q}_{2}(x)<q_{1}(x)$ for $x \in\left(x^{*}, \frac{\lambda_{2}-1}{c_{2}}\right)$ and every solution in $R_{2}$ will enter $\operatorname{int}\left(Q_{4}\left(E^{*}\right)\right)$ in finite time steps by [7, Theorem 4]. To show every solution in $R_{2}$ converges to $E_{0}$, it is enough to consider those solutions in $\operatorname{int}\left(Q_{4}\left(E^{*}\right)\right)$. Notice that $E^{*} \ll_{K}$ $\left(x_{0}, y_{0}\right) \leq_{K} F\left(x_{0}, y_{0}\right)$ for any $x_{0} \in\left(x^{*}, \frac{\lambda_{2}-1}{c_{2}}\right)$ and $y_{0}=\frac{q_{1}\left(x_{0}\right)+\hat{q}_{2}\left(x_{0}\right)}{2}$. Let $(x, y) \in \operatorname{int}\left(Q_{4}\left(E^{*}\right)\right)$ be arbitrary. Then we can find $\left(x_{0}, y_{0}\right)$, where $x_{0} \in\left(x^{*}, \frac{\lambda_{2}-1}{c_{2}}\right)$ and $y_{0}=\frac{q_{1}\left(x_{0}\right)+\hat{q}_{2}\left(x_{0}\right)}{2}$, such that $\left(x_{0}, y_{0}\right) \leq_{K}(x, y)$. As a result, $E^{*} \ll_{K} F^{n}\left(x_{0}, y_{0}\right) \leq_{K} F^{n}(x, y)$ for $n=0,1,2, \ldots$ and $F^{n}(x, y)$ cannot converge to $E^{*}$. Thus solutions in $R_{2}$ converge to $E_{0}$ and case 3 holds.

(iii) implies that (2.4) has two interior steady states $E_{i}^{*}=\left(x_{i}^{*}, y_{i}^{*}\right), i=1,2, x_{1}^{*}<x_{2}^{*}$. Then $E_{1}^{*} \ll_{K} E_{2}^{*}$ and it can be readily verified that $E_{1}^{*}$ is locally asymptotically stable and $E_{2}^{*}$ is a saddle point. Indeed, $q_{1}^{\prime}\left(x_{1}^{*}\right)<\hat{q}_{2}^{\prime}\left(x_{1}^{*}\right), q_{1}^{\prime}\left(x_{2}^{*}\right)>\hat{q}_{2}^{\prime}\left(x_{2}^{*}\right)$, and $q_{2}^{\prime}\left(y_{i}^{*}\right)=\frac{1}{\hat{q}_{2}^{\prime}\left(x_{i}^{*}\right)}$ for $i=1,2$. Therefore at $E_{2}^{*}$, we have by (2.19), $p(1)=\left(q_{1}^{\prime}\left(x_{2}^{*}\right) q_{2}^{\prime}\left(y_{2}^{*}\right)-1\right) b c<0$ and $E_{2}^{*}$ is a saddle point. At $E_{1}^{*}$, we have $p(1)=\left(q_{1}^{\prime}\left(x_{1}^{*}\right) q_{2}^{\prime}\left(y_{1}^{*}\right)-1\right) b c>0$ and $\operatorname{det} J\left(E_{1}^{*}\right)=a d-b c<a d<1$ by (2.16)-(2.18). Therefore, $E_{1}^{*}$ is locally asymptotically stable. The stable manifold $W_{2}^{s}$ of $E_{2}^{*}$ is a strictly increasing $C^{1}$-curve by [7]. Since $Q_{1}\left(E_{2}^{*}\right)$ has neither steady state nor 2-cycle solutions, $W_{2}^{s}$ is unbounded in $Q_{1}\left(E_{2}^{*}\right)$ and $W_{2}^{s}$ intersects with the nonnegative $y$-axis. Therefore $W_{2}^{s}$ separates $\mathbb{R}_{+}^{2}$ into two positively invariant regions $R_{i}, i=1$, 2, with $E_{1}^{*} \in R_{1}$ and $E_{0} \in R_{2}$. The remaining assertion follows and case 4 holds. 
If $m>u$, then $y=q_{1}(x)$ may not be monotone decreasing. However, it can easily be shown that the $x$ component of any interior steady state satisfies a polynomial of degree 3 . To this end, let

$$
H(x)=\left(\hat{q}_{2}(x)-q_{1}(x)\right)(x-u)(m+x) .
$$

Then

$$
\begin{aligned}
H(x)= & \left(1-c_{1} c_{2}\right) x^{3}+\left[\left(1-c_{1} c_{2}\right)(m-u)+c_{1}\left(\lambda_{2}-1\right)+1-\lambda_{1}\right] x^{2} \\
& +\left[(m-u)\left(c_{1}\left(\lambda_{2}-1\right)+1\right)-m u\left(1-c_{1} c_{2}\right)\right] x-m u\left[c_{1}\left(\lambda_{2}-1\right)+1\right]
\end{aligned}
$$

and

$$
\begin{aligned}
H^{\prime}(x)= & 3\left(1-c_{1} c_{2}\right) x^{2}+2\left[\left(1-c_{1} c_{2}\right)(m-u)+c_{1}\left(\lambda_{2}-1\right)+1-\lambda_{1}\right] x \\
& +\left[(m-u)\left(c_{1}\left(\lambda_{2}-1\right)+1\right)-m u\left(1-c_{1} c_{2}\right)\right] .
\end{aligned}
$$

Any positive solution $x^{*}$ of $H(x)=0$ satisfying $x^{*}<\min \left\{\bar{x}, \frac{\lambda_{2}-1}{c_{2}}\right\}$ will result in an interior steady state of (2.4). Moreover, if $\left(x_{0}, y_{0}\right)$ is an interior steady state of (2.4), then

$$
H^{\prime}\left(x_{0}\right)=\left(\hat{q}_{2}^{\prime}\left(x_{0}\right)-q_{1}^{\prime}\left(x_{0}\right)\right)\left(x_{0}-u\right)\left(m+x_{0}\right) .
$$

In the following, we separate our discussion into the cases of $c_{1} c_{2}<1, c_{1} c_{2}>1$, and $c_{1} c_{2}=1$, respectively.

Let $1-c_{1} c_{2}>0$. Observe that $\lim _{x \rightarrow \infty} H(x)=\infty, \lim _{x \rightarrow-\infty} H(x)=-\infty, H(u)<0$ and $H(x)$ has at most two critical points. The asymptotic dynamics of (2.4) is given below.

Theorem 3.3 Let $\frac{\lambda_{2}-1}{c_{2}}>u, m>u$, and $c_{1} c_{2}<1$. The following statements hold for (2.4).

(a) Let $\bar{x}<\frac{\lambda_{2}-1}{c_{2}}$. Then (2.4) has at least one interior steady state.

(i) Case 2 holds if there exist a unique $x^{*} \in(u, \bar{x})$ such that $H(x)<0$ on $\left(u, x^{*}\right)$ and $H(x)>0$ on $\left(x^{*}, \bar{x}\right)$.

(ii) Case 6 holds if there exist $z_{i}, i=1,2, u<z_{1}<z_{2}<\bar{x}$ such that $H^{\prime}\left(z_{1}\right)=0=H^{\prime}\left(z_{2}\right)$ and $H\left(z_{1}\right)>0>H\left(z_{2}\right)$.

(iii) Case 5 holds if there exist $z_{i}, i=1,2, u<z_{1}<z_{2}<\bar{x}$ such that $H^{\prime}\left(z_{1}\right)=0=H^{\prime}\left(z_{2}\right)$ and either $H\left(z_{1}\right)=0>H\left(z_{2}\right)$ or $H\left(z_{1}\right)>0=H\left(z_{2}\right)$.

(b) Let $\bar{x}>\frac{\lambda_{2}-1}{c_{2}}$.

(i) Case 1 holds if $H(x)<0$ on $\left(u, \frac{\lambda_{2}-1}{c_{2}}\right)$.

(ii) Case 4 holds if there exists $z \in\left(u, \frac{\lambda_{2}-1}{c_{2}}\right)$ such that $H^{\prime}(z)=0$ and $H(z)>0$.

(iii) Case 3 holds if there exists $x^{*}=z \in\left(u, \frac{\lambda_{2}-1}{c_{2}}\right)$ such that $H^{\prime}(z)=0 \neq H^{\prime \prime}(z)$ and $H(z)=0$.

Proof (a) Since $\bar{x}<\frac{\lambda_{2}-1}{c_{2}}, E_{0}$ is a saddle point with stable manifold lying on the nonnegative $x$-axis. Moreover, $H(\bar{x})>0$ and $H(u)<0$ imply $H(x)=0$ has at least one solution lying between $u$ and $\bar{x}$. Hence (2.4) has at least one interior steady state. The assumption of (i) implies that (2.4) has a unique interior steady state $E^{*}=\left(x^{*}, y^{*}\right)$ and $H^{\prime}\left(x^{*}\right)>0$. It can be shown that $E^{*}$ is locally asymptotically stable since $\hat{q}_{2}^{\prime}\left(x^{*}\right)>q_{1}^{\prime}\left(x^{*}\right)$ by (3.5). Therefore, $E^{*}$ is globally asymptotically stable in $\Gamma$ and case 2 holds. 
If the assumptions of (ii) hold, then $H(x)=0$ has three solutions $x_{i}^{*}, i=1,2,3$, with $u<$ $x_{1}^{*}<z_{1}<x_{2}^{*}<z_{2}<x_{3}^{*}<\bar{x}$. Hence (2.4) has three interior steady states $E_{i}^{*}=\left(x_{i}^{*}, y_{i}^{*}\right), i=1,2,3$, with $E_{1}^{*} \ll_{K} E_{2}^{*} \ll_{K} E_{3}^{*}$. Moreover, $H^{\prime}\left(x_{i}^{*}\right)>0$ for $i=1,3$ and $H^{\prime}\left(x_{2}^{*}\right)<0$ imply

$$
\hat{q}_{2}^{\prime}\left(x_{i}^{*}\right)>q_{1}\left(x_{i}^{*}\right) \quad \text { for } i=1,3 \quad \text { and } \quad \hat{q}_{2}^{\prime}\left(x_{2}^{*}\right)<q_{1}\left(x_{2}^{*}\right)
$$

Therefore, $E_{i}^{*}, i=1,3$, are locally asymptotically stable and $E_{2}^{*}$ is a saddle point by (2.16)(2.19). The stable manifold $W_{2}^{s}$ of $E_{2}^{*}$ is a strictly increasing $C^{1}$-curve that extends indefinitely in $Q_{1}\left(E_{2}^{*}\right)$ and intersects the nonnegative $y$-axis. It follows that $W_{2}^{s}$ separates $\mathbb{R}_{+}^{2}$ into two positively invariant regions $R_{1}$ and $R_{2}$ such that $E_{1}^{*} \in R_{1}$ and $E_{3}^{*} \in R_{2}$. It can then be shown similarly by Theorem 2.3 that $E_{1}^{*}$ and $E_{2}^{*}$ are globally asymptotically stable in $R_{1}$ and $R_{2}$, respectively, i.e., case 6 holds.

(iii) is the critical case of (ii). Notice that if $H(x)>0$ for $x>z_{1}$ and sufficiently close to $z_{1}$, then by the assumption on $z_{2}$ there exist $z^{*} \in\left(z_{1}, z_{2}\right)$ such that $H^{\prime}\left(z^{*}\right)=0$, which contradicts to the fact that $H(x)$ has at most two critical points. Therefore, $z_{1}$ is a non-degenerate critical point and hence (2.4) has two interior steady states $E_{i}^{*}=\left(x_{i}^{*}, y_{i}^{*}\right), i=1,2$, where $u<x_{1}^{*}=z_{1}<z_{2}<x_{2}^{*}<\bar{x}$. Since $H^{\prime}\left(x_{1}^{*}\right)=0, E_{1}^{*}$ is non-hyperbolic by (2.19) and (3.5). The other eigenvalue of $J\left(E_{1}^{*}\right)$ is $\operatorname{det} J\left(E_{1}^{*}\right)=a d-b c<1$ with corresponding eigenvectors not parallel to the coordinate axes. On the other hand, $H^{\prime}\left(x_{2}^{*}\right)>0$ implies that $E_{2}^{*}$ is locally asymptotically stable. The stable manifold $W_{1}^{s}$ is a strictly increasing $C^{1}$-curve that intersects the nonnegative $y$-axis and extends indefinitely in $Q_{1}\left(E_{1}^{*}\right)$. It follows that $W_{1}^{s}$ separates $\mathbb{R}_{+}^{2}$ into two positively invariant regions $R_{1}$ and $R_{2}$ with $E_{2}^{*} \in R_{2}$. Consequently, solutions with initial conditions in $R_{1}$ converge to $E_{1}^{*}$ by a similar proof as that of Theorem 3.2(b)(ii), and $E_{2}^{*}$ is globally asymptotically stable in $R_{2}$, i.e., case 5 holds. The other critical case can be shown similarly.

(b) Since $\bar{x}>\frac{\lambda_{2}-1}{c_{2}}, E_{0}$ is locally asymptotically stable. Also $H(u)<0$ and $H\left(\frac{\lambda_{2}-1}{c_{2}}\right)<0$. If $H(x)<0$ on the interval $\left(u, \frac{\lambda_{2}-1}{c_{2}}\right)$, then $(2.4)$ has no interior steady state and $E_{0}$ is globally asymptotically stable by Theorem 2.3 . This proves (i).

(ii) Note first that such a $z$ given in the assumption is unique and hence (2.4) has two interior steady states $E_{i}^{*}=\left(x_{i}, y_{i}\right), i=1,2$, where $E_{1}^{*} \ll_{K} E_{2}^{*}$. It is straightforward to verify that $E_{1}^{*}$ is locally asymptotically stable and $E_{2}^{*}$ is a saddle point. The stable manifold $W_{2}^{s}$ of $E_{2}^{*}$ is a strictly increasing $C^{1}$-curve that intersects the nonnegative $y$-axis and is unbounded in $Q_{1}\left(E_{2}^{*}\right)$. The remaining assertion follows. The assumption of (iii) implies that the existence of a unique interior steady state $E^{*}=\left(x^{*}, y^{*}\right)$, where $x^{*}=z$, which is, moreover, non-hyperbolic since $H^{\prime}\left(x^{*}\right)=0$. However, the other eigenvalue of $J\left(E^{*}\right)$ is $\operatorname{det} J\left(E^{*}\right)=a d-b c<a d<1$ with corresponding eigenvectors not parallel to the coordinate axes. The remaining proof is similar to the proof (ii) of Theorem 3.2(b).

Figure 1(a)-(f) provides the graph of $y=H(x)$ for $c_{1} c_{2}<1$. In Figure 1(a), $m=2, u=0.9$, $c_{1}=c_{2}=0.5, \lambda_{1}=20.5$, and $\lambda_{2}=20$. Then $\bar{x}=18.45<\frac{\lambda_{2}-1}{c_{2}}=38$ and $H(x)=0$ has a unique positive solution in $(u, \bar{x})$. When the constant stocking $u$ is decreased to $u=0.3$, then $\bar{x}=$ $17.74<\frac{\lambda_{2}-1}{c_{2}}=38$ and $H(x)=0$ has three positive solutions in $(u, \bar{x})$ as shown in Figure 1(b). If $u=0.4$, then $\bar{x}=17.86<\frac{\lambda_{2}-1}{c_{2}}=38$ and $H(x)=0$ has two feasible positive solutions where the smaller solution is a critical point of $H(x)$. See Figure 1(c).

Let $m=2, u=0.9, c_{1}=c_{2}=0.5, \lambda_{1}=12.5$, and $\lambda_{2}=6$. Then $\bar{x}=10.48>\frac{\lambda_{2}-1}{c_{2}}=10$ and $y=H(x)$ lies below the horizontal axis as shown in Figure 1(d). If $u$ is decreased to $u=0.15$ 


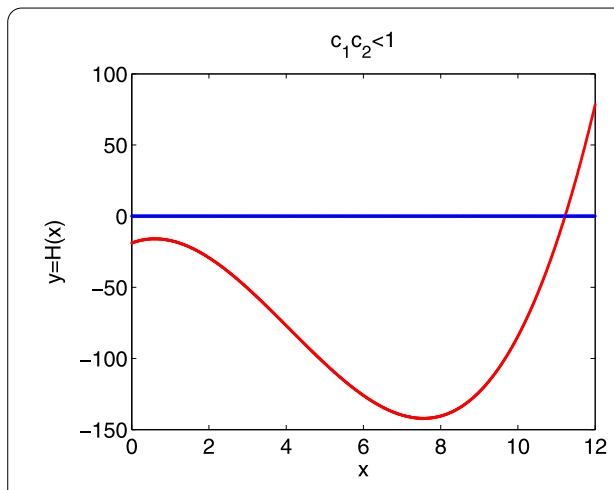

(a)

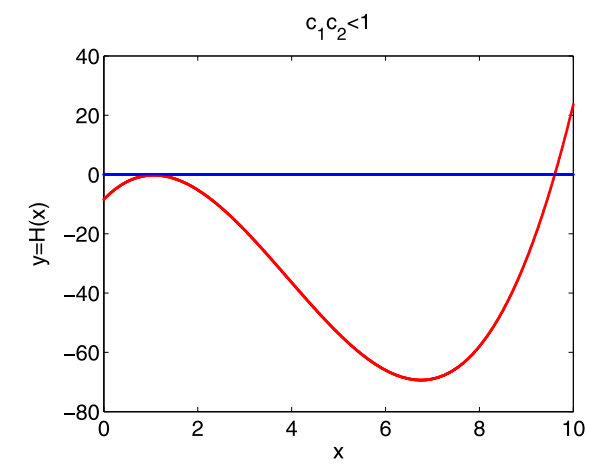

(c)

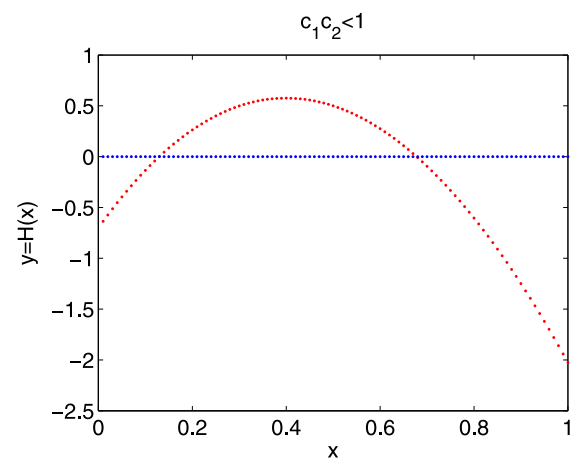

(e)

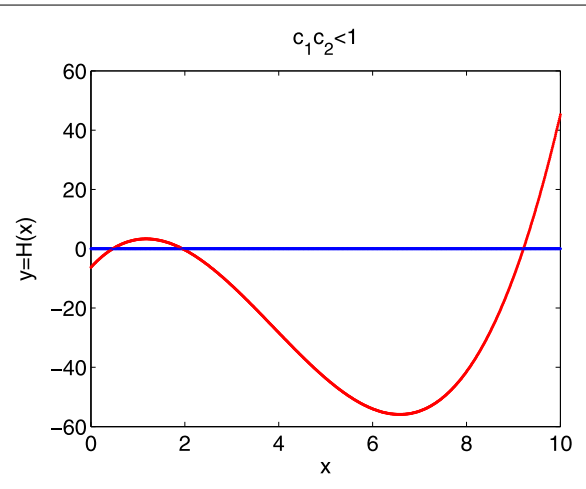

(b)

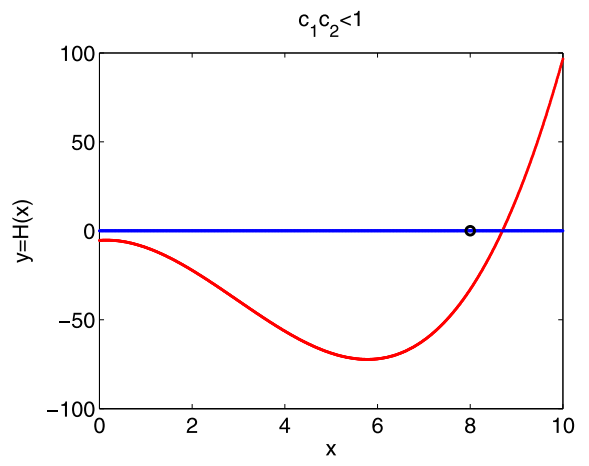

(d)

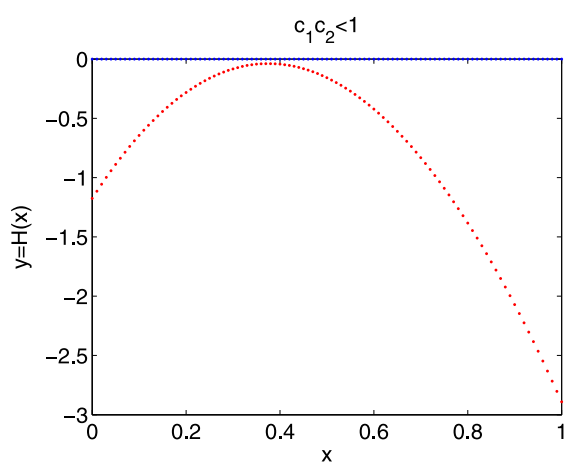

(f)

Figure 1 Plots (a)-(c) illustrate the number of solutions of $H(x)=0$ in Theorem 3.3(a) cases (i), (ii), and (iii), respectively, and (d)-(f) are for Theorem 3.3(b) cases (i), (ii), and (iii), respectively. The point $\min \left\{\bar{x}, \frac{\lambda_{2}-1}{c_{2}}\right\}$ on the $x$-axis is not plotted if it is large.

with $\lambda_{1}=13.5$, then $\bar{x}=10.51>\frac{\lambda_{2}-1}{c_{2}}=10$ and $H(x)=0$ has two positive solutions within the feasible region as shown in Figure 1(e). If $u$ is increased slightly to $u=0.16789$, then $\bar{x}=10.53>\frac{\lambda_{2}-1}{c_{2}}=10$ and $H(x)=0$ has a unique positive solution within the feasible region as shown in Figure 1(f).

Consider the case where $1-c_{1} c_{2}<0$. Then $\lim _{x \rightarrow \infty} H(x)=-\infty, \lim _{x \rightarrow-\infty} H(x)=\infty$, $H(u)<0$ and $H(x)=0$ has at least one negative solution. Hence, $H(x)=0$ can have at most two positive solutions. The asymptotic dynamics of (2.4) is given below. 
Theorem 3.4 Let $\frac{\lambda_{2}-1}{c_{2}}>u, m>u$, and $c_{1} c_{2}>1$. The following statements hold for (2.4).

(a) If $\bar{x}<\frac{\lambda_{2}-1}{c_{2}}$, then case 2 holds.

(b) Let $\bar{x}>\frac{\lambda_{2}-1}{c_{2}}$.

(i) Case 1 holds if $H(x)<0$ on $\left(u, \frac{\lambda_{2}-1}{c_{2}}\right)$.

(ii) Case 4 holds if there exists $z \in\left(u, \frac{\lambda_{2}-1}{c_{2}}\right)$ such that $H^{\prime}(z)=0$ and $H(z)>0$.

(iii) Case 5 holds if the critical point $z$ in (ii) is such that $H(z)=0 \neq H^{\prime \prime}(z)$.

Proof (a) By the assumption, we have $H(\bar{x})>0, \lim _{x \rightarrow \infty} H(x)=-\infty$, and $\lim _{x \rightarrow-\infty} H(x)=$ $\infty$. Therefore $H(x)=0$ has a unique positive solution $x^{*}$ in $(u, \bar{x})$ with $H^{\prime}\left(x^{*}\right)>0$. The remaining assertion follows.

(b) Notice that $H\left(\frac{\lambda_{2}-1}{c_{2}}\right)<0$ and $E_{0}$ is asymptotically stable. Since $H(x)=0$ has at least one negative root, $H(x)=0$ can have at most two positive roots in $\left(u, \frac{\lambda_{2}-1}{c_{2}}\right)$. (i) If $H(x)<0$ on $\left(u, \frac{\lambda_{2}-1}{c_{2}}\right)$, then $H(x)=0$ has no positive solution on $\left(u, \frac{\lambda_{2}-1}{c_{2}}\right)$ and (2.4) has no interior steady state. It follows that $E_{0}$ is globally asymptotically stable and case 1 holds.

(ii) By the assumption, $H(x)=0$ has two positive solutions $x_{i}^{*}, i=1,2$, where $u<x_{1}^{*}<z<$ $x_{2}^{*}<\frac{\lambda_{2}-1}{c_{2}}$. Hence (2.4) has two interior steady states $E_{i}^{*}=\left(x_{i}^{*}, y_{i}^{*}\right), i=1,2, E_{1}^{*} \ll_{K} E_{2}^{*}$. It can be verified that $E_{1}^{*}$ is asymptotically stable and $E_{2}^{*}$ is a saddle point. The remaining of the proof is similar to the proof of Theorem 3.3(b)(ii) and case 4 holds. (iii) is a critical case of (ii) and $H(x)=0$ implies $x_{1}^{*}=z$. System (2.4) has two interior steady states $E_{i}^{*}=\left(x_{i}^{*}, y_{i}^{*}\right)$, $i=1,2$, where $E_{1}^{*}$ is non-hyperbolic and $E_{2}^{*}$ is asymptotically stable. The remaining proof is similar to the proof of Theorem 3.3(a)(iii).

Remark 2 The condition in Theorem 3.3(a)(ii) is a sufficient condition for the existence of three interior steady states. The condition is feasible since $H(x)$ is a polynomial of degree 3 so that it is possible for $y=H(x)$ to have two critical points in $(u, \bar{x})$. Similarly, conditions stated in Theorem 3.3(b) and Theorem 3.4 are sufficient conditions for the existence of one or two interior steady states. There are possibly other different sufficient conditions.

In Figure 2(a), we let $m=2.0, u=0.9, c_{1}=2.1, c_{2}=0.5, \lambda_{1}=5$, and $\lambda_{2}=6$. Then $\bar{x}=3.28<$ $\frac{\lambda_{2}-1}{c_{2}}=10$ and $H(x)=0$ has a unique positive solution in $(u, \bar{x})$. If we change $\lambda_{1}$ and $\lambda_{2}$ to $\lambda_{1}=10$ and $\lambda_{2}=3$, then $\bar{x}=8.01>\frac{\lambda_{2}-1}{c_{2}}=4$ and $H(x)=0$ has no positive solution as shown in Figure 2(b). If we let $u=0.2$, then $\bar{x}=7.01>\frac{\lambda_{2}-1}{c_{2}}=4$ and $H(x)=0$ has two positive solutions as shown in Figure 2(c). If we let $u=0.4$, then $\bar{x}=7.31>\frac{\lambda_{2}-1}{c_{2}}=4 . H(x)=0$ has a unique positive solution $x^{*}$ and the horizonal axis is tangent to the curve $y=H(x)$ at $x^{*}$. See Figure 2(d).

If $c_{1} c_{2}=1$, then $H(x)$ becomes a quadratic function,

$$
H(x)=\left[c_{1}\left(\lambda_{2}-1\right)+1-\lambda_{1}\right] x^{2}+\left[(m-u)\left(c_{1}\left(\lambda_{2}-1\right)+1\right)\right] x-m u\left[c_{1}\left(\lambda_{2}-1\right)+1\right] .
$$

Observe that $c_{1}\left(\lambda_{2}-1\right)+1>0$ by (2.7). Let

$$
\Delta=c_{1}\left(\lambda_{2}-1\right)+1-\lambda_{1}
$$

If $\Delta>0$, then $H(x)$ is a concave up parabola with a negative vertical intercept and a negative $x$ coordinate at the vertex. On the other hand, if $\Delta<0$, then $H(x)$ is a concave down 


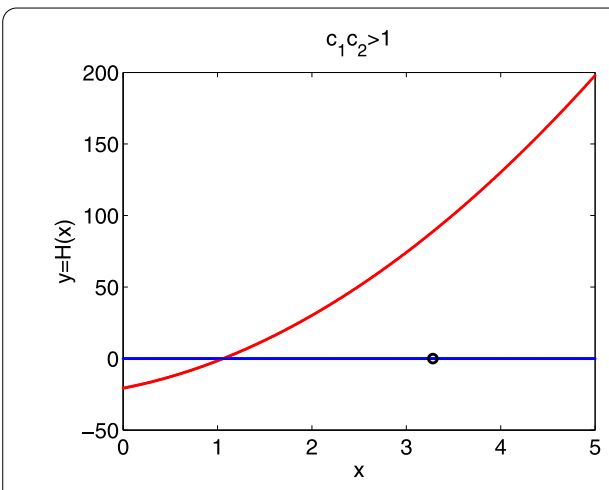

(a)

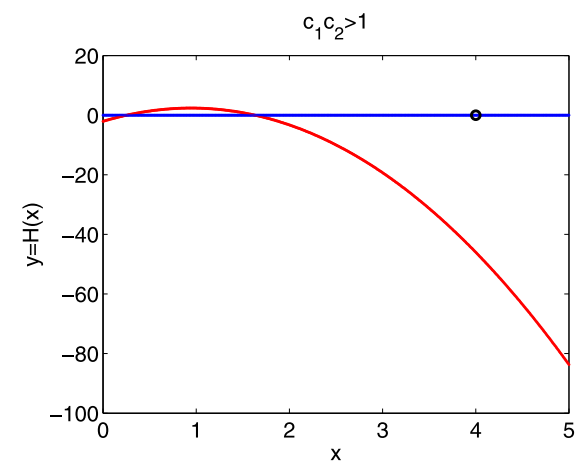

(c)

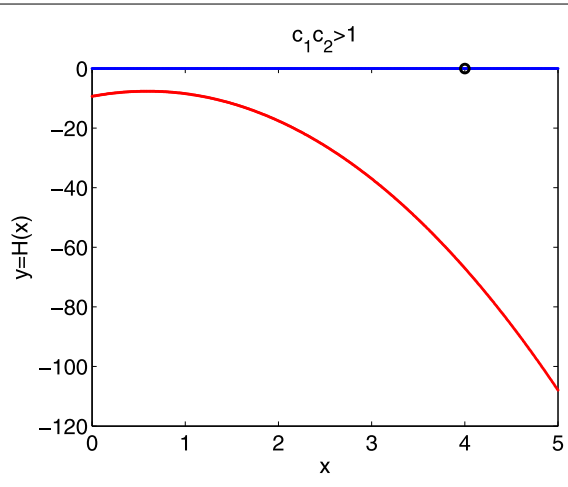

(b)

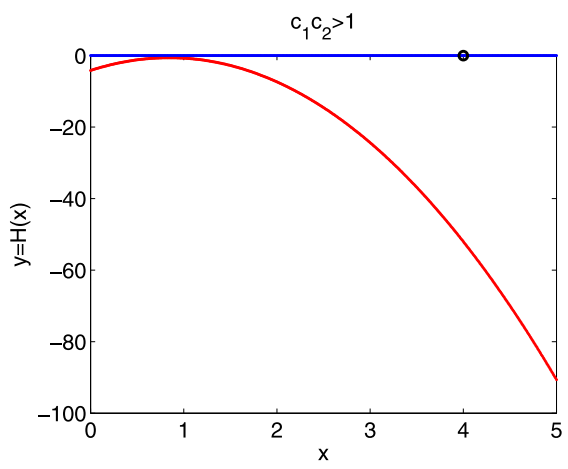

(d)

Figure 2 Plots (a)-(d) demonstrate Theorem 3.4(a) and (b) cases (i)-(iii), respectively. The point marked on the $x$ axis is the value of $\min \left\{\bar{x}, \frac{\lambda_{2}-1}{c_{2}}\right\}$.

parabola with a negative vertical intercept and a positive $x$ coordinate at the vertex,

$$
\hat{x}=\frac{(m-u)\left(c_{1}\left(\lambda_{2}-1\right)+1\right)}{-2 \Delta}>0 .
$$

When $\Delta=0$, then $H(x)$ is a linear function with a negative $y$-intercept.

The asymptotic dynamics of system (2.4) when $c_{1} c_{2}=1$ is summarized below. Since the proof of Theorem 3.5 is similar to the proofs of Theorems 3.2-3.4, we omit the proof.

Theorem 3.5 Let $\frac{\lambda_{2}-1}{c_{2}}>u, m>u$, and $c_{1} c_{2}=1$. The following statements hold for (2.4).

(a) Let $\Delta>0$.
(i) If $\bar{x}<\frac{\lambda_{2}-1}{c_{2}}$, then case 2 holds.
(ii) If $\bar{x}>\frac{\lambda_{2}-1}{c_{2}}$, then case 1 holds.

(b) Let $\Delta<0$.

(i) If $\bar{x}<\frac{\lambda_{2}-1}{c_{2}}$, then case 2 holds.

(ii) Let $\bar{x}>\frac{\lambda_{2}-1}{c_{2}}$. If $\hat{x} \in\left(u, \frac{\lambda_{2}-1}{c_{2}}\right)$ and $H(\hat{x})>0$, then case 4 holds. If $\hat{x} \in\left(u, \frac{\lambda_{2}-1}{c_{2}}\right)$ and $H(\hat{x})=0$, then case 3 holds. If either $\hat{x} \notin\left(u, \frac{\lambda_{2}-1}{c_{2}}\right)$ or if $\hat{x} \in\left(u, \frac{\lambda_{2}-1}{c_{2}}\right)$ and $H(\hat{x})<0$, then case 1 holds.

(c) Let $\Delta=0$.

(i) If $\bar{x}<\frac{\lambda_{2}-1}{c_{2}}$, then case 2 holds.

(ii) If $\bar{x}>\frac{\lambda_{2}-1}{c_{2}}$, then case 1 holds. 


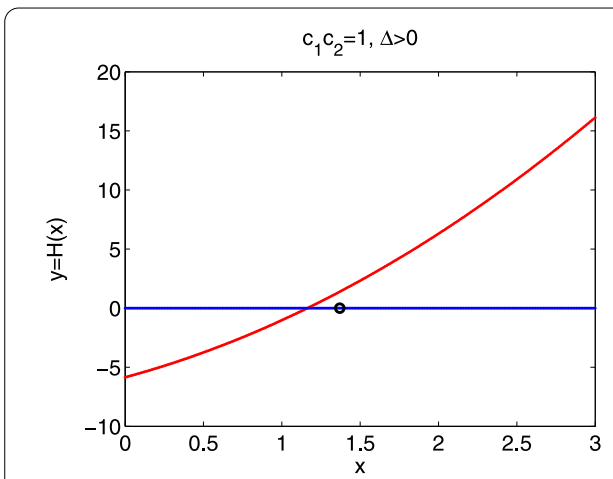

(a)

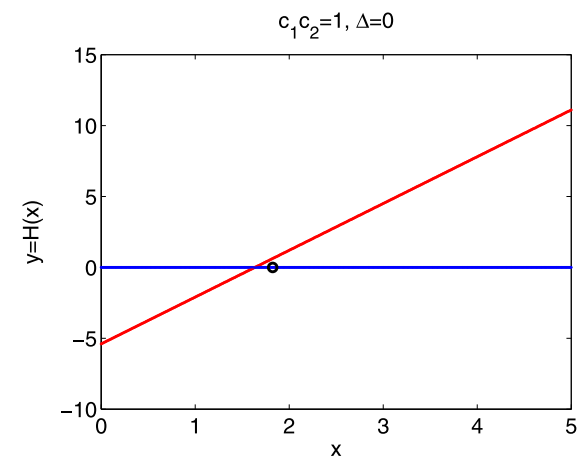

(c)

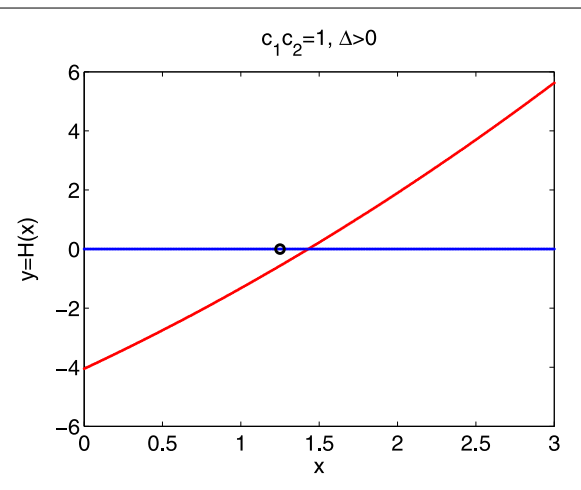

(b)

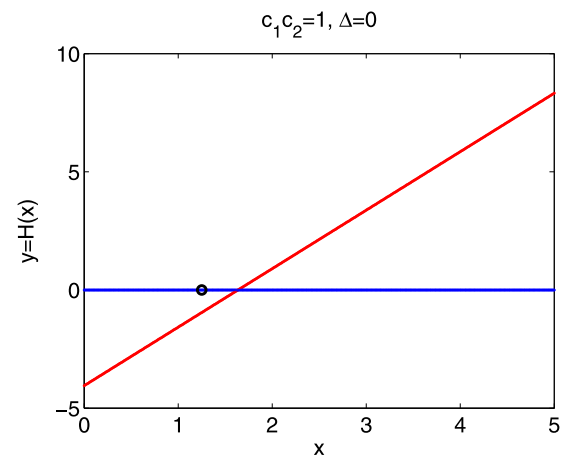

(d)

Figure 3 The figure illustrates the number of solutions of $H(x)=0$ in Theorem 3.5 for $\Delta>0$ in (a)-(b) and $\boldsymbol{\Delta}=\mathbf{0}$ in (c)-(d). The point marked on the $x$ axis is the value of $\min \left\{\bar{x}, \frac{\lambda_{2}-1}{c_{2}}\right\}$.

Figures 3 and 4 provide the graph $y=H(x)$ for the case $c_{1} c_{2}=1$. The parameter values $m=2, u=0.9, c_{1}=0.5$, and $c_{2}=2$ are fixed. In Figure $3(\mathrm{a}), \lambda_{1}=2$ and $\lambda_{2}=5.5$ so that $\Delta=$ $1.25>0$ and $\bar{x}=1.37<\frac{\lambda_{2}-1}{c_{2}}=2.25$. The equation $H(x)=0$ has a unique positive solution in $(u, \bar{x})$. In Figure 3(b), $\lambda_{2}$ is decreased to $\lambda_{2}=3.5$ such that $\bar{x}=1.37>\frac{\lambda_{2}-1}{c_{2}}=1.25$. In this case, the positive solution of $H(x)=0$ exceeds $\frac{\lambda_{2}-1}{c_{2}}$ and (2.4) has no interior steady state. In order to obtain $\Delta=0$, we let $\lambda_{1}=c_{1}\left(\lambda_{2}-1\right)+1$ and vary $\lambda_{2}$. In Figure 3(c), we have $\lambda_{2}=5.5$. Then $\bar{x}=1.97<\frac{\lambda_{2}-1}{c_{2}}=2.25$ and $H(x)=0$ has a unique solution in $(u, \bar{x})$. In Figure $3(\mathrm{~d})$, we let $\lambda_{2}=3.5$. Then $\bar{x}=1.47>\frac{\lambda_{2}-1}{c_{2}}=1.25$ and the unique positive solution of $H(x)=0$ does not lie inside the interval $\left(u, \frac{\lambda_{2}-1}{c_{2}}\right)$.

In Figure 4(a), we use $\lambda_{1}=5.2$ and $\lambda_{2}=8.5$, then $\Delta=-0.45<0, \bar{x}=3.45<\frac{\lambda_{2}-1}{c_{2}}=3.75$ and $H(x)=0$ has a unique solution lying in $(u, \bar{x})$. In Figure 4(b), where $\lambda_{1}=3$ and $\lambda_{2}=$ 3.5 , and we have $\Delta=-0.75<0$ and $\bar{x}=1.82>\frac{\lambda_{2}-1}{c_{2}}=1.25$. Then $H(x)<0$ on the feasible interval so that $H(x)=0$ has no positive solution. If we let $\lambda_{1}=10.6$ and $\lambda_{2}=17$, then $\Delta=-1.6<0$ and $\bar{x}=8.61>\frac{\lambda_{2}-1}{c_{2}}=8.0$. In this case, the horizontal axis is tangent to the curve $y=H(x)$ at its vertex as shown in Figure 4(c). When $\lambda_{2}$ is increased to $\lambda_{2}=18$, then $\Delta=-1.1<0$ and $\bar{x}=8.61>\frac{\lambda_{2}-1}{c_{2}}=8.5$. The equation $H(x)=0$ has two positive solutions in the interval $\left(u, \frac{\lambda_{2}-1}{c_{2}}\right)$. See Figure $4(\mathrm{~d})$.

Remark 3 In this study we focus on the case when the $x$ population has only one interior steady state. If there are three boundary steady states, then since the boundary $x$-dynamics 


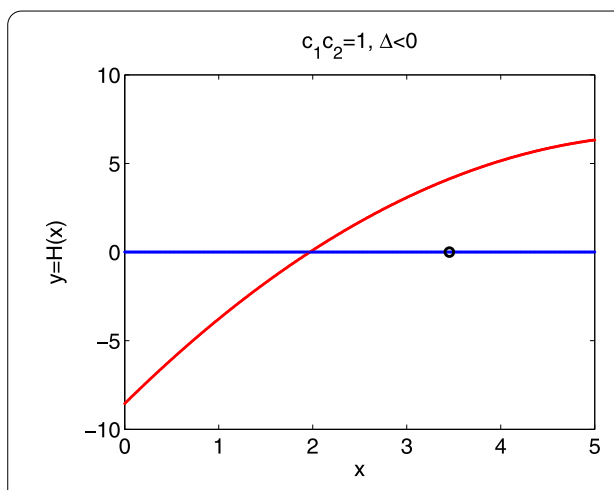

(a)

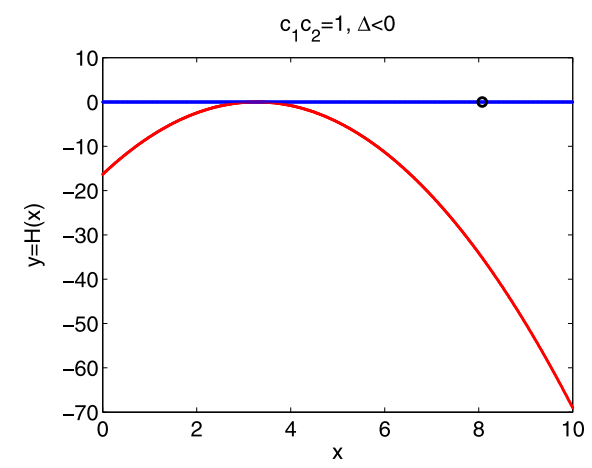

(c)

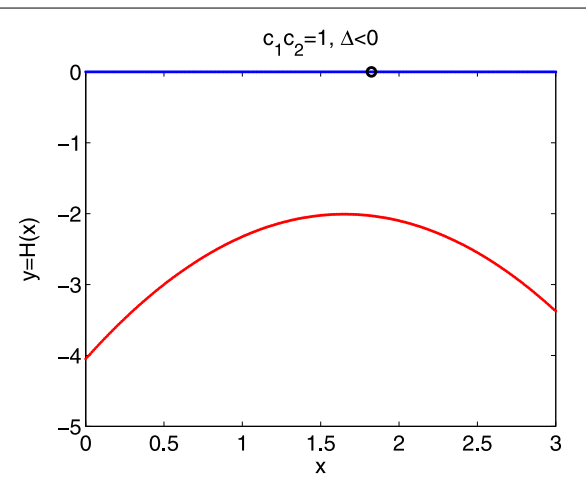

(b)

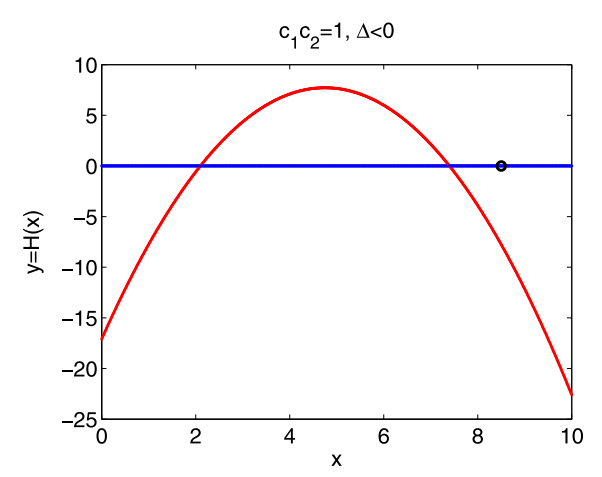

(d)

Figure 4 (a)-(d) illustrate the number of solutions of $H(x)=0$ in Theorem 3.5(b) where $\Delta<0$. The point marked on the $x$ axis is the value of $\min \left\{\bar{x}, \frac{\lambda_{2}-1}{c_{2}}\right\}$.

is completely known, we can apply a similar analysis by separating $\mathbb{R}_{+}^{2}$ into several positively invariant regions instead of only two regions and the corresponding asymptotic dynamics can also be obtained.

\section{Discussion and conclusions}

Allee effects are phenomena in biology which arise when per-capita growth rates increase with increasing population level up to a maximum and then decline thereafter due to intraspecific competition [1]. The phenomena have received considerable attention recently due to fragmentation of habitats, invasion of exotic species, and biological control of pest, etc. [2], all involving small populations. When a population is subject to Allee effects, it is well known that there exists a critical population level below which the population will go extinct [2]. Therefore, the population is in danger of extinction if its population size or density is small. Consequently, constant levels of the external populations are frequently added to the resident populations per generation in order to conserve the endangered populations [3].

In [5], Elaydi and Yakubu considered the following competition system governed by the Ricker equations:

$$
\begin{aligned}
& x_{1}(t+1)=x_{1}(t) e^{-p_{1}-q_{1}\left(x_{1}(t)+x_{2}(t)\right)}+\alpha x_{1}(t), \\
& x_{2}(t+1)=x_{2}(t) e^{-p_{2}-q_{2}\left(x_{1}(t)+x_{2}(t)\right)},
\end{aligned}
$$


where the parameters $p_{i}, q_{i}, i=1,2$, are positive, and $\alpha \in(0,1)$ denotes stocking. System (4.1) has been studied by Yakubu previously in [16] and [17]. Yakubu obtained parameter regimes for the occurrence of an attracting 2-cycle and conjectured that the 2-cycle is globally stable. The study in [5] disproved this conjecture and concluded that a 2-cycle cannot be the ultimate life-history of evolution of all population sizes [5]. Since (4.1) is based on the Ricker equations which are nonmonotone and system (2.4) is monotone, the dynamics of (4.1) is more complex than the dynamics of (2.4), where every solution of (2.4) converges to a steady state.

AlSharawi and Rhouma [4] on the other hand studied the following system of $n$ populations:

$$
x_{i}(t+1)=\frac{b_{i} x_{i}(t)}{1+\sum_{j=1}^{n} c_{i j} x_{j}(t)}+h_{i}, \quad 1 \leq i \leq n,
$$

where $b_{i}$ and $c_{i j}$ are positive constants, $1 \leq i, j \leq n$, and $h_{i}$ denotes either harvesting or stocking depending on whether $h_{i}<0$ or $h_{i}>0,1 \leq i \leq n$. In particular, AlSharawi and Rhouma proved that if stocking occurs for all $n$ populations, i.e., $h_{i}>0$ for $1 \leq i \leq n$, then the unique interior steady state is globally asymptotically stable so that coexistence occurs for all positive populations [4].

Kulenović and Nurkanović [6] studied a special case of (4.2), namely $n=2$ with $h_{2}=0$ and $h_{1}=h>0$, i.e., system (2.2). It is proved in [6] that (2.2) has at most two interior steady states and in such a case the smaller interior steady state is a saddle point. The stable manifold of the saddle point then separates $\mathbb{R}_{+}^{2}$ into two positively invariant regions such that solutions in either region converge to the corresponding steady state, i.e., one region of solutions converges to the coexisting steady state, while the $x_{2}$ population goes extinct in the other region. That is, case 4 of Table 1 holds for system (2.2).

In this investigation, we also consider only two competing populations where one of the populations is subject to Allee effects and is also under stocking. The resulting system (2.4) has a richer dynamics than that of the model (2.2) with no Allee effects. Indeed, Table 1 summarizes different asymptotic behavior of model (2.4). In addition to case 4 , it is possible for system (2.4) to have three interior steady states where the middle interior steady state is a saddle point. The stable manifold of the saddle point separates $\mathbb{R}_{+}^{2}$ into two positively invariant regions such that solutions in each of the regions converge to the corresponding interior steady state. Therefore, when stocking is implemented for the population with Allee effects, it can promote the coexistence of the two competing populations.

We next discuss the effects of constant stocking $u$ on system (2.3). First, notice that those steady states of (2.3) on the nonnegative $y$-axis all disappear for (2.4). Therefor stocking of the $x$ population prevents the population from extinction. Secondly, model (2.3) always has a region $\Gamma$, depending on the Allee threshold of the $x$ population, such that the $y$ population out-competes the $x$ population if the initial population distributions lie in $\Gamma$. See Theorem 2.7 of [6]. In particular, the $y$ population will drive the $x$ population to extinction if the $x$ population is small. With stocking of the $x$ population, the region $\Gamma$ disappears so that the $y$ population will not out-compete the $x$ population when the $x$ population is small as long as the constant stocking $u$ satisfies the assumption of Proposition 2.1. Moreover, if the stocking $u$ is large, then the $x$ population will drive the $y$ population to extinction 
even when the $x$ population is subject to Allee effects as demonstrated in Theorem 3.1. As a consequence, large stocking can reverse competition outcomes.

The most important effect of the stocking is that system (2.4) can have three interior steady states. Without stocking, system (2.3) has at most two interior steady states where the smaller one is a saddle point and the other steady state is asymptotically stable. One region of the solutions converges to the steady state on the positive $y$-axis so that the $x$ population is driven to extinction when the population is subject to Allee effects but there is no stocking. When system (2.4) has three interior steady states, then the middle interior steady state is a saddle point while the other two interior steady states are asymptotically stable. See Theorem 3.3(a)(ii). The stable manifold of the saddle point separates $\mathbb{R}_{+}^{2}$ into two positively invariant regions such that solutions in each of these regions converge to the corresponding interior steady state. Therefore, for this parameter regime, given in Theorem 3.3(a)(ii), both competing populations can coexist as an interior steady state, where the magnitude of the population sizes depends on the initial populations.

Finally, system (2.4) can have a unique global asymptotic stable interior steady state as given in case 2 of Table 1 . This global stability signifies that dynamical behavior of (2.4) is no longer initial condition dependent. The dependence on the initial conditions can be viewed as a hallmark of the Allee effects and stocking of the population can erase this hallmark and can promote a robust coexistence between the two populations.

Competing interests

The authors declare that they have no competing interests.

Authors? contributions

All authors contributed equally to the writing of this paper. All authors read and approved the final manuscript.

Author details

${ }^{1}$ Institute of Mathematics, Academia Sinica, Taipei, 10617, Taiwan. ${ }^{2}$ Department of Mathematics and Statistics, Texas Tech University, Lubbock, TX 79409-1042, USA.

\section{Acknowledgements}

We thank one of the referees for helpful comments and suggestions.

Received: 2 May 2014 Accepted: 19 December 2014 Published online: 14 January 2015

References

1. Allee, WC: The Social Life of Animals. William Heinemann, London (1938)

2. Courchamp, F, Berec, L, Gascoigne, J: Allee Effects in Ecology and Conservation. Oxford University Press, New York (2008)

3. Nock, C, Ovenden, J, Butler, G, Wooden, I, Moore, A, Baverstock, J: Population structure, effective population size and adverse effects of stocking in the endangered Australian eastern freshwater cod Maccullochella ikei. J. Fish Biol. 78, 303-321 (2011)

4. AlSharawi, Z, Rhouma, M: Coexistence and extinction in a competitive exclusion Leslie/Gower model with harvesting and stocking. J. Differ. Equ. Appl. 15, 1031-1053 (2009)

5. Elaydi, S, Yakubu, A: Global stability of cycles: Lotka-Volterra competition model with stocking. J. Differ. Equ. Appl. 8, 537-549 (2002)

6. Kulenović, M, Nurkanović, M: Global behavior of a two-dimensional competitive system of difference equations with stocking. Math. Comput. Model. 55, 1998-2011 (2012)

7. Kulenović, M, Merino, O: Invariant manifolds for competitive discrete systems in the plane. Int. J. Bifurc. Chaos 20, 2471-2486 (2010)

8. Smith, HL: Planar competitive and cooperative difference equations. J. Differ. Equ. Appl. 3, 335-357 (1998)

9. Jang, SR-J: Competitive exclusion and coexistence in a Leslie-Gower competition model with Allee effects. Appl. Anal. 92, 1527-1540 (2013)

10. Cushing, JM, Levarge, S, Chitnis, N, Henson, S: Some discrete competition models and the competitive exclusion principle. J. Differ. Equ. Appl. 10, 1139-1151 (2004)

11. Leslie, PH, Gower, JC: The properties of a stochastic model for two competing species. Biometrika 45, 316-330 (1958)

12. Schreiber, S: Allee effects, extinctions, and chaotic transients in simple population models. Theor. Popul. Biol. 64, 201-209 (2003)

13. Allen, L: An Introduction to Mathematical Biology. Prentice Hall, New York (2006)

14. Jang, SR-J: Allee effects in a discrete-time host-parasitoid model. J. Differ. Equ. Appl. 12, 165-181 (2006) 
15. Wiggins, S: Introduction to Applied Nonlinear Dynamical Systems and Chaos, 2nd edn. Springer, New York (2003)

16. Yakubu, $A$ : The effects of planting and harvesting on endangered species in discrete competitive systems. Math. Biosci. 126, 1-20 (1995)

17. Yakubu, A: A discrete competitive system with planting. J. Differ. Equ. Appl. 4, 213-214 (1998)

Submit your manuscript to a SpringerOpen ${ }^{\circ}$ journal and benefit from:

- Convenient online submission

- Rigorous peer review

- Immediate publication on acceptance

- Open access: articles freely available online

- High visibility within the field

- Retaining the copyright to your article 